\title{
La représentation politique des minorités linguistiques
}

Une analyse comparée de trois partis ethnorégionalistes d'Italie

Political representation of linguistic minorities: a comparative analysis of three ethno-regionalist parties in Italy

\section{Carlo Pala et Giulia Sandri}

\section{(2) OpenEdition}

\section{Journals}

\section{Édition électronique}

URL : http://journals.openedition.org/conflits/18041

DOI : 10.4000/conflits. 18041

ISSN : $1777-5345$

Éditeur :

CCLS - Centre d'études sur les conflits lilberté et sécurité, L'Harmattan

\section{Édition imprimée}

Date de publication : 26 décembre 2010

Pagination : $97-122$

ISBN : 978-2-296-56047-5

ISSN : 1157-996X

Référence électronique

Carlo Pala et Giulia Sandri, «La représentation politique des minorités linguistiques », Cultures \& Conflits [En ligne], 79-80 | Automne/Hiver 2010, mis en ligne le 26 mai 2012, consulté le 30 mars 2021. URL : http://journals.openedition.org/conflits/18041; DOI : https://doi.org/10.4000/conflits. 18041 


\title{
La représentation politique des minorités linguistiques
}

\author{
Une analyse comparée de trois partis ethnorégionalistes \\ $\mathrm{d}^{\prime}$ Italie 1
}

\section{Carlo PALA et Giulia SANDRI}

Carlo Pala est Post-Doc en Science Politique à l'Université de Cagliari. Ses intérêts de recherche portent sur les partis ethnorégionalistes européens, les régionalismes breton et sarde, les élections primaires en Italie et la politique locale. En français, il a publié avec Giulia Sandri "L'impact du processus de régionalisation sur le système politique et partisan italien ", dans Pilet J.-B. et al., L'absence de partis nationaux : menace ou opportunité ?, aux Éditions de l'Université de Bruxelles.

Giulia Sandri est doctorante à l'Université libre de Bruxelles. Ses intérêts de recherche portent sur les partis ethnorégionalistes européens, le régionalisme en Italie, la participation politique et l'adhésion partisane en particulier. En français, elle a publié "Les partis ethnorégionalistes et les conséquences de la participation au pouvoir : le cas de l'Union Valdôtaine ", Fédéralisme - Régionalisme, vol. 8 : $2008, n^{\circ} 2$.

\section{Les origines des mobilisations ethnolinguistiques en Italie}

T a question de la protection de la diversité linguistique en Italie est souvent Lliée avec la protection des minorités ethno-territoriales. On souligne notamment comment, pour étouffer l'aspiration des peuples d'ethnie non italienne à s'organiser politiquement de façon autonome, l'État central « a coupé » les langues minoritaires ${ }^{2}$. Déjà, pendant la période fasciste, puis après la seconde Guerre mondiale, les protestations des régions périphériques, où se concentrent les minorités ethnolinguistiques du pays, semblaient mener à une demande généralisée d'autonomie, voire d'indépendance de leurs territoires.

1. Bien que cet article soit issu d'un travail commun, Carlo Pala a rédigé les paragraphes 1, 2 et 5 ; Giulia Sandri les paragraphes 3, 4 et 6.

2. Salvi S., Le lingue tagliate, Milan, Rizzoli, 1975, pp. 161-166. 
Mouvements et partis politiques à tendances séparatiste, irrédentiste ou rattachiste apparaissent en Sicile et en Sardaigne et, dans une moindre mesure, en Trentin, en Haut-Adige/Sud-Tyrol, en Vallée d'Aoste dès le début du XXe siècle (dans certains cas, plus tôt encore). L'existence d'une distance significative entre l'identité culturelle des Italiens et le sentiment d'appartenance nationale naît de façon évidente au moment de l'unification du pays, achevée en 1861, quand émergent les premiers mouvements autonomistes en Vallée d'Aoste et en Sardaigne. La question du Trentin-Haut-Adige se pose depuis l'armistice de 1918 et oscille pendant des décennies entre des moments de normalisation, avec des protestations populaires, et des moments de radicalisation, avec l'apparition des premiers attentats et des bombes (particulièrement entre les années 1956 et 1967) jusqu'au début des années quatre-vingt-dix. Celle de Trieste survient au moment de l'annexion dans les années cinquante et perdure dans l'agenda politique local, national et parfois international jusqu'aux années quatre-vingt.

La Constitution de 1948 (article 6), dispose que les minorités linguistiques italiennes sont protégées par la loi. Mais à l'exception du français en Vallée d'Aoste et de l'allemand en Haut-Adige - langues qui bénéficient de la même parité légale que l'italien dans les statuts des régions correspondantes - aucune protection n'a été allouée à d'autres langues parlées sur le territoire italien au niveau national pendant les cinquante dernières années (comme le sarde et le frioulan). Cinquante ans après l'adoption de la Constitution, l'Italie a (re)découvert sa différenciation culturelle interne en termes de langue et d'ethnie lors d'un nouvel essor des mobilisations ethnolinguistiques, ainsi qu'à l'occasion de l'émergence de la Ligue du Nord (LN) en tant qu'acteur politique. L'émergence de la LN en termes de compétition électorale et de participation au gouvernement, tant au niveau régional pendant les années quatrevingt qu'au niveau national au début des années quatre-vingt-dix, a imposé à nouveau la question ethno-territoriale sur l'agenda politique national après des décennies d'une relative latence du clivage centre - périphérie. Ce clivage était resté présent au niveau national des années soixante aux années quatrevingt, mais marginal, tant dans le discours politique des partis que dans les mobilisations sociales, et relégué aux aspects spécifiques de gestion de la question de Trieste et du Sud-Tyrol. Il faut donc noter que, si les questions d'ethno-régionalismes n'ont jamais quitté l'agenda politique italien, le succès électoral et politique de la LN depuis les années quatre-vingt-dix leur a redonné une saillance qui avait été fortement mitigée pendant les décennies précédentes.

Il faut toutefois prêter attention à la différenciation fondamentale entre, d'une part, la montée en puissance de la Ligue du Nord depuis les années quatre-vingt-dix et, d'autre part, le problème plus prégnant des minorités linguistiques (reconnues ou non) sur le territoire italien. La LN est en effet souvent classée parmi les partis territoriaux, c'est-à-dire représentant un territoire spé- 
cifique à travers des stratégies politiques de type régionaliste mais n'étant pas bâtis sur des clivages ethniques ou linguistiques ${ }^{3}$. L'idéologie fédéraliste de la LN est fondée sur des critères davantage économiques que politiques et la construction de l'identité nationale de la Padania (le territoire du Nord-Est de l'Italie, étalé sur plusieurs régions, que la LN est censée représenter) n’a pas été autant mobilisée que dans les autres partis régionalistes italiens, faute d'éléments ethnolinguistiques présents au Val d'Aoste, au Frioul, dans le SudTyrol et en Sardaigne. Pour ces raisons, la classification de la LN parmi les partis ethnorégionalistes est parfois contestée ${ }^{4}$. Si la LN prétend défendre les intérêts de l'ethnie nordiste (la « Nordnazione » dans son jargon), cette dernière relève d'une « invention de la tradition 5 » qui ne possède pas d'ancrage dans la réalité sociale, culturelle et historique des territoires concernés, dans aucune des acceptions du concept d'ethnicité identifiées par la littérature ${ }^{6}$. Néanmoins, il existe historiquement, dans les années soixante et soixante-dix, des liens entre les réseaux préalables à ce qui deviendra la Ligue du Nord et les autonomistes de l'Union Valdôtaine (UV) et du Südtiroler Volkspartei (SVP). Par exemple, Bruno Salvadori, un des principaux idéologues de l'UV, a entretenu des rapports très étroits dans les années soixante-dix avec Umberto Bossi et est à la base des premières formulations de la pensée fédéraliste de la $\mathrm{LN}^{7}$. D'ailleurs, les acteurs de la mobilisation ethnolinguistique en Italie ont toujours combiné la requête de politiques de défense de la langue avec la requête d'une forte intervention financière de l'État et de systèmes de protection fiscale pour les régions intéressées ${ }^{8}$. La question linguistique en Italie est donc fortement liée, aujourd'hui, à des requêtes de décentralisation culturelle aussi bien qu'à des requêtes plus strictement économiques. La langue est de plus en plus souvent instrumentalisée à des fins politiques.

Jusqu'à présent et à l'exception de rares cas, la régionalisation italienne a été réalisée par le haut, sous impulsion de l'État central ${ }^{9}$. Ceci a entraîné un manque d'intérêt spécifique pour la protection des langues minoritaires, la loi

3. Pilet J.-B., De Waele J.-M. et Jaumain S. (ed), L'absence de partis nationaux : menace ou opportunité ?, Bruxelles, Éditions de l’Université de Bruxelles, 2009, pp. 97-124.

4. McDonnell D., "A Weekend in Padania: Regionalist Populism and the Lega Nord", Politics 26(2), 2006, pp. 126-132 ; McDonnell D., "Beyond the the Radical Right Straitjacket", Politics, 27(2), 2007, pp. 123-126 ; Zaslove A. “Alpine Populism, Padania and Beyond”, Politics, 27(1), 2007, pp. 64-68.

5. Hobsbawm, Eric J., Ranger T. O., The Invention of tradition, Cambridge, Cambridge University Press, 1983

6. Schmidke O., Politics of Identity. Ethnicity, Territory and the Political Opportunity Structure in Modern Italian Society, Sinzheim, Pro Universitate, 1996, pp. 10-22 ; Wolff S., Cordell K., Ethnopolitical Encyclopaedia of Europe, Basingstoke, Palgrave, 2004 ; Huysseune M., Modernità e secessione. Le scienze sociali e il discorso politico della Lega Nord, Roma, Carocci, 2004.

7. Stévénin F., Bruno Salvadori, Aosta, Musumeci, 2005, pp. 189-198.

8. Toso F., Le minoranze linguistiche in Italia, Bologne, Il Mulino, 2008.

9. Sandri G. et Pala C., "L’impact du processus de régionalisation sur le système politique et partisan italien ", in Pilet J.-B., De Waele J.-M. et Jaumain S. (ed), L'absence de partis nationaux : menace ou opportunité ?, Bruxelles, Éditions de l’Université de Bruxelles, 2009, pp. 97124. 
482/1999 constituant le seul exemple de législation en la matière. La question des minorités linguistiques a été reléguée à une position de problème résiduel. Bien que l'Italie ait signé la Charte Européenne des langues minoritaires, l’État rendait en fait très difficile son application.

En effet, l'adoption des statuts régionaux spéciaux en 1948 (pour la Sardaigne, la Sicile, la Vallée d'Aoste, le Frioul, le Trentin-Haut-Adige), " octroyés " par l'État italien afin d'apaiser la mobilisation identitaire des périphéries historiques ${ }^{10}$, est liée, dans le cas de la Vallée d'Aoste et du HautAdige, à la nécessité de contrebalancer les mouvements irrédentistes apparus pendant la guerre et appuyés au niveau international respectivement par la France et l'Autriche. Dans le cas de la Sardaigne, les raisons sous-jacentes à l'octroi de l'autonomie étaient moins liées à des considérations ethnonationales qu'à des motivations de développement entretenues par la situation d'isolement géographique et de retard économique ${ }^{11}$. L'ampleur de l'avantage fiscal pour la Vallée d'Aoste et le Trentin Haut-Adige découlant de l'autonomie statutaire est considérable. Par exemple, dans le cas valdôtain, neuf dixièmes de l'impôt prélevé sur le territoire est attribué à la région. Les transferts fiscaux sont de la même portée dans le cas du Haut-Adige. Ces deux régions représentent des cas assez uniques en Europe d'achats de la loyauté d'un groupe linguistique minoritaire par un État.

La Ligue du Nord a toutefois soulevé, à partir des années quatre-vingtdix, le problème des ethnies minoritaires et des territoires périphériques au niveau de l'État central. Auparavant, ceci n'avait pas été possible en raison de la faible représentation électorale à l'échelle nationale des mouvements politiques représentant les minorités ethnolinguistiques historiques (francophones en Vallée d'Aoste, germanophones en Sud-Tyrol, slovènophones et frioulans en Frioul, sardophones en Sardaigne). En fait, si ces acteurs sont souvent hégémoniques à l'échelle locale et régionale, leur force relative dans la compétition électorale au niveau national reste limitée en raison de la concentration territoriale de leur soutien électoral. Etant donné ce contexte politique et historique ${ }^{12}$, il serait intéressant d'examiner les acteurs politiques qui ont joué un rôle prééminent dans la représentation des groupes ethnolinguistiques mentionnés ci-dessus, c'est-à-dire les partis ethnorégionalistes actifs dans les régions où les minorités ethnolinguistiques sont concentrées. Néanmoins, l'attention de la science politique dédiée au phénomène ethno-nationaliste, ethnorégionaliste et ethnolinguistique et aux conflits correspondants en Italie est restée marginale jusqu'à la dernière décennie ${ }^{13}$. Les acteurs du conflit centre-périphérie qui se positionnent également par rapport à la protection d'une

10. Nevola G. (ed.), Altre Italie. Identità nazionale e regioni a statuto speciale, Carocci, Rome, 2003, p. XVI-XVII.

11. Ibid., XXIV.

12. Ibidem.

13. Nevola G. (ed.), Op. cit.; Tronconi F., I partiti etnoregionalisti, Bologne, Il Mulino, 2009. 
langue minoritaire restent en fait en marge de l'intérêt des chercheurs. On analysera donc ici le parcours identitaire de l'Union Valdôtaine (UV) pour la minorité française en Vallée d'Aoste, le Südtiroler Volkspartei (SVP) pour celle allemande en Sud-Tyrol-Haut-Adige et le Partito Sardo d'Azione (PSdAZ) pour la Sardaigne et la langue sarde. Il s'agit de trois exemples représentant les trois minorités ethnolinguistiques historiques, dont nous avons analysé les trois partis principaux. Après avoir esquissé dans quelle mesure le clivage ethnolinguistique s'enracine dans un conflit social primitif, réactivé lors de chaque élection dans les trois régions sélectionnées, on verra aussi, en examinant les documents internes de ces partis, le positionnement idéologique par rapport aux concepts de langue, ethnie et territoire des trois partis qui prétendent représenter les intérêts des trois minorités ethnolinguistiques francophone, germanophone et sardophone.

\section{Les partis ethnorégionalistes italiens}

La particularité du cas italien apparaît, tout d'abord, dans la faible dimension électorale de ces formations politiques au niveau national, et, ensuite, par la forte présence des représentants des conflits ethnolinguistiques nouveaux (ou bien tout à fait inventés). Les chercheurs affirment que l'Italie peut difficilement être considérée comme un véritable État-Nation ${ }^{14}$. Le phénomène ethnorégionaliste en Italie, donc, acquiert une pertinence et une forme particulières, inhérentes aux dimensions des forces politiques concernées et différentes, par exemple, des partis ethnorégionalistes en France ${ }^{15}$.

Le contexte institutionnel, en particulier, axé sur l'existence de cinq régions à statut spécial, a permis aux partis ethnorégionalistes italiens de se consolider. On peut ainsi considérer comme centrale la position du SVP dans l'arène politique de la province autonome de Bolzano. Le SVP est un véritable parti prédominant dans le sens sartorien ${ }^{16} \mathrm{du}$ terme. Les rôles historiques de l'UV et du PSdAZ sont plus nuancés, bien que le premier soit plus stable en termes de participation au pouvoir que le second. Ces partis sont désormais devenus un point de repère électoral important pour les électeurs, bien qu'ils n'avancent que des requêtes très spécifiques au niveau du changement de l'arrangement institutionnel et de l'aménagement territorial de l'État ou de protections de la minorité linguistique ${ }^{17}$. À l'exception des partis indépendan-

14. Rusconi G.E., Se cessiamo di essere una nazione. Tra etnodemocrazie regionali e cittadinanza europea, Bologne, Il Mulino,1993.

15. Gómez-Reino Cachafeiro M. et Pasquier R., «Les partis régionalistes et nationalistes en Europe du Sud. Une comparaison Espagne, France et Italie », Pôle Sud, 20, Mai 2004, p. 5 ; Fournis Y. et Pasquier R., «Le développement des partis régionalistes en Europe de l'Ouest. De la crise de l'État-Nation à la transformation du clivage territorial ", in Fougerouse J. (ed.), L'État régional, une nouvelle forme d'État? Un exemple de recomposition territoriale en Europe et en France, Bruxelles, Bruylant, 2008, pp. 183-199.

16. Sartori G., «The Typology of Party Systems : Proposal for Improvement », dans Allardt E. et Rokkan S. (ed), Mass Politics, New York, Free Press, pp. 332-337. 
tistes sardes et d'autres petits partis rattachistes ou irrédentistes, les partis ethnorégionalistes italiens ont généralement avancé des requêtes d'autonomie politico-institutionnelle.

En effet, le clivage ethnolinguistique ne dérive pas seulement de l'existence du discours différencié mis en avant par les trois partis sélectionnés. Ce clivage s'enracine plutôt dans un conflit social fort ancré qui existe depuis des siècles dans les trois régions en question et qui est réactivé lors de chaque élection. Afin d'examiner la «force » de ce clivage il faut donc étudier les résultats électoraux effectifs de ces partis. Cela nous permet aussi de présenter une mise en contexte des trois partis, en esquissant leur poids relatif dans la compétition électorale et le système de partis tant au niveau régional qu'au niveau national. D'une part, les tableaux 1, 2 et 3 (annexe 1) montrent deux formations politiques, l'UV et le SVP, qui apparaissent comme prédominantes dans leurs systèmes de partis régionaux respectifs. Leur force électorale au niveau régional est relativement stable (dans le cas du SVP) ou plus oscillante (dans le cas de l'UV) mais reste significative tout au long de la période examinée. De plus, le SVP et l'UV bénéficient chacun de dispositifs électoraux spéciaux prévus pour les deux régions autonomes quant à l'allocation de sièges au Parlement à Rome. Ces deux partis ont ainsi réussi à presque monopoliser la représentation externe de la région au parlement national. En revanche, le parcours électoral du PSdAZ est jalonné d'incidents, avec des résultats très différents d'une élection à l'autre et un poids politique plus limité dans le système des partis régionaux. D’autre part, le faible impact électoral de ces formations politiques au niveau national est évident (l'UV et le PSdAZ représentent en moyenne $0,1 \%$ des voix au niveau national, le SVP environ 0,5\%) et lié principalement à la concentration territoriale de leur support électoral dans les trois régions respectives.

La confirmation de l'existence du clivage en question est apportée non seulement par le résultat électoral de certains partis, mais aussi par le fait que l'identité ethnolinguistique, avec l'identité historique, économique et culturelle des régions en conflit avec le centre, constitue une superstructure toujours en mesure de mobiliser politiquement les habitants de ces régions. Dans certaines régions italiennes le problème est inversé, c'est-à-dire qu'il y a une surabondance de partis ethnorégionalistes. Le phénomène de la multiplication de ces partis produit une situation qu'on peut qualifier de reproductibilité : des

17. Pour quelques références, on pourrait lire entièrement les travaux suivants : Newman $S$., «Ethnoregionalist parties : a comparative perspective », Regional Politics and Policy, 2, 1994, pp. 28-66 ; Türsan H. et De Winter L. (ed), Regionalist parties in Western Europe, Londres, Routledge, 1998, pp. 204-247 ; Delwit P. (ed), Les partis régionalistes en Europe. Des acteurs en développement ?, Bruxelles, Editions de l’Université de Bruxelles, 2005, pp. 51-84 ; De Winter L., Gómez-Reino Cachafeiro M. et Lynch P. (ed), Autonomist parties in Europe : Identity, Politics, and the Revival of the territorial Cleavage, Barcelone, Institut de Ciències Polítiques i Socials, 2006, vol. 2, pp. 247-271 ; Tronconi F., I partiti etnoregionalisti, Bologne, Il Mulino, 2009, pp. 161-183. 
forces politiques régionalistes naissent sans posséder d'éléments distinctifs par rapport à celles déjà existantes. La langue et le concept d'ethnie restent deux fondements de l'élaboration programmatique et idéologique comme de l'action pratique des partis considérés. Les trois communautés linguistiques historiques analysées ici représentent les trois cas les plus significatifs de minorités linguistiques mobilisées politiquement, tant du point de vue des dimensions et de la durée des mobilisations que de la cristallisation et de l'enracinement du clivage centre-périphérie. Les minorités germanophone et francophone présentent la caractéristique d'être minorisées dans le contexte national, bien qu'elles relèvent d'un groupe linguistique majoritaire dans d'autres pays européens, tandis que la troisième est doublement minorisée, au niveau national et au niveau européen, en parlant une langue véhiculaire unique (le sarde).

\section{L'analyse des partis et des groupes ethnolinguistiques sélectionnés}

Cette étude croise deux aspects principaux des mobilisations ethnonationalistes 18 en Europe : la structuration de l'identité culturelle des minorités ethnolinguistiques, d'une part, et la nature de leur représentation politique, d'autre part. L'étude vise donc à répondre à deux principaux axes de recherche : 1) analyser le rôle de la langue en tant que vecteur identitaire dans les communautés linguistiques minoritaires ; 2 ) identifier le rôle de la langue comme objectif politique des organisations politiques ethnorégionalistes sur le territoire considéré. La langue est en fait un vecteur identitaire très fort dans certains cas, mais très faible dans d'autres.

Afin d'analyser les facteurs favorisant le rôle de la langue en tant que «catalyseur » identitaire à l'intérieur des mouvements ethnonationalistes, la théorie des clivages développée par Seymour M. Lipset et Stein Rokkan ${ }^{19}$ est appliquée ici, et en particulier la théorie du clivage centre-périphérie de Stein Rokkan et Derek Urwin 20. Les multiples fractures engendrées par le clivage centre-périphérie sont décrites en termes d'interaction d'éléments géographiques, culturels, économiques et politico-institutionnels. Rokkan et Urwin réservent aussi une position privilégiée dans leur cadre théorique aux ressources culturelles, de type religieux (le groupe minoritaire fonde son identité sur l'appartenance à une religion), linguistique (le groupe minoritaire possède une identité spécifique non seulement culturelle mais aussi linguistique), ou historique. La mobilisation politique des acteurs territoriaux, y compris les

18. Les termes d'ethnonationalisme, ethnorégionalisme, mobilisation ethnolinguistique et ethnoterritoriale sont utilisés ici comme synonymes.

19. Lipset S. M. et Rokkan S., Party Systems and Voter Alignments : Cross-National Perspectives, New York, The Free Press, 1967, pp. 1-63.

20. La théorie utilisée ici est notamment développée tout au long de ces deux ouvrages : Rokkan S. et Urwin D.W., Economy, territory, identity: politics of West European peripheries, Londres, Sage, 1983 ; Rokkan S. et Urwin D.W., The Politics of Territorial Identity: Studies in European Regionalism, Londres, Sage, 1982. 
acteurs ethnolinguistiques, est donc définie comme le résultat d'une intégration territoriale des États incomplète ou manquante. Les inégalités et les asymétries qui persistent à l'intérieur des États-nations européens créent des facteurs potentiels de mobilisation (des catalyseurs) que les entrepreneurs politiques peuvent exploiter pour la recherche et l'agrégation du consensus ${ }^{21}$.

Selon Pietro Grilli di Cortona 22, les dimensions analytiques à retenir, outre les quatre dimensions identifiées par Rokkan et Urwin, sont démographiques (le groupe minoritaire est concentré dans une région ou une zone territoriale spécifiques), institutionnelles (l'identité du groupe minoritaire se fonde sur la mémoire historique commune des formes d'autonomie politique et institutionnelle obtenues dans le passé) et internationales (le groupe ethnique minoritaire est le correspondant ethnique ou ethnic kin d'un groupe national, constituant la majorité de la population d'un autre État ou kin state). L'Italie étant un État homogène en terme de religion, seules les dimensions linguistiques, démographiques, internationales et politico-institutionnelles sont intégrées dans l'analyse.

La seconde question de recherche porte sur le rôle de la langue dans l'idéologie des organisations politiques ethnorégionalistes. Le paradigme des partis ethnorégionalistes développé par De Winter et Türsan en 1998 est aussi appliqué dans cette étude. Cette approche définit les partis ethnorégionalistes en tant qu' « entrepreneurs ethniques 23 » qui revendiquent non seulement une réorganisation politique de la structure étatique territoriale, mais se basent également sur l'existence de clivages ethniques dans la région concernée. La catégorie de "parti ethnorégionaliste » intègre donc la notion d'etbnos, de communauté unie par des liens culturels, linguistiques et identitaires, à la notion de concentration territoriale au niveau infra-étatique ${ }^{24}$.

Cette définition établit trois principaux éléments constitutifs des organisations qui représentent politiquement les mouvements ethnolinguistiques : la langue, l'ethnie et le territoire. L'analyse de la déclinaison de ces trois éléments à l'intérieur du discours politique et de l'idéologie de cette «famille » de partis permet d'identifier l'évolution du rôle de la langue en tant qu'objectif politique. Quatre facteurs principaux affectent la structure idéologique des partis ethnorégionalistes : l'évolution des rapports de l'autorité infra-étatique avec l'État central, l'évolution de la structure démographique des territoires concernés, l'évolution des performances électorales et du statut compétitif des partis analysés et leur participation au pouvoir.

21. Tronconi F., op. cit.

22. Grilli di Cortona P., Stati, Nazioni e Nazionalismi in Europa, Bologne, Il Mulino, 2003.

23. Türsan H. et De Winter L. (ed), op. cit., pp. 5-7.

24. Gellner E., Nations and Nationalism, Ithaca, Cornell University Press, 1983 ; Connor W., Ethnonationalism: The Quest for Understanding, Princeton, Princeton University Press, 1994 ; Brown D., Contemporary Nationalism Civic. Ethnocultural and Multicultural Politics, Londres, Routledge, 2000. 
L'étude analyse de façon comparée la représentation politique des groupes ethnolinguistiques actifs en Italie. Les trois cas (plus larges et mieux représentés politiquement, possédant des statuts légaux et internationaux très différents) sont étudiés par rapport aux autres groupes ethnolinguistiques italiens (slovènes, ladins, catalans, grecs, albanais, etc.). Pour tracer l'évolution des traits idéologiques des partis considérés, l'étude utilise une approche méthodologique qualitative dans l'analyse des discours politiques développés par l'UV, le SVP et le PSdAZ pendant les soixante dernières années (19482009) 25. La dimension temporelle de l'analyse peut apparaitre ample, néanmoins l'objectif d'identifier les modifications intervenues dans le discours politique des trois formations analysées nécessite une analyse diachronique suffisamment développée. Il est important de souligner en particulier que les trois partis examinés ici étaient déjà actifs dans l'arène politique et électorale régionale, voire nationale, au moment des premières élections régionales en 1948. L'examen des programmes des trois partis pour les élections régionales, nationales et européennes et du contenu de leurs documents internes (résolutions finales et rapports des congrès du parti) constituent le terrain de cette étude. Le corpus empirique est constitué donc par une cinquantaine de documents de longueur variée, de quatre ou cinq pages à plus de cinquante pages ${ }^{26}$.

25. Entre 1947 et 1949 les premières élections des parlements régionaux des régions à statut spécial furent organisées.

26. Il s'agit des programmes électoraux des trois partis aux élections européennes de 1979, 1984, 1989, 1994, 1999, 2004, 2009, ainsi que les programmes aux élections nationales de 1948, 1953, 1958 (pour l'UV, l'analyse des programmes électoraux nationaux commence en 1958, qui correspond à la première fois où le parti se présente avec une formation distincte) , 1963, 1968, 1973, 1976, 1979, 1983, 1987, 1992, 1994, 1996, 2001, 2006, 2008 et aux élections régionales respectives $(1949,1954,1959,1963,1968,1973,1978,1983,1988,1993,1998,2003,2008$ pour l'UV ; 1948, 1952, 1956, 1960, 1964, 1968, 1973, 1978, 1983, 1988, 1993, 1998, 2003, 2008 pour le SVP ; 1949, 1953, 1957, 1961, 1965, 1969, 1974, 1979, 1984, 1989, 1994, 1999, 2004, 2009 pour le PSdAZ). Ces documents sont soit disponibles en ligne soit ont été obtenus auprès des organes centraux des trois partis. Nous avons analysé également les résolutions finales et rapports de congrès des trois partis. Pour l'UV, nous avons analysé les résolutions de tous les congrès à parti du premier congrès national de 1976 ; pour le PSdAZ, les résolutions de tous les congrès depuis 1921 ; pour le SVP, en raison de problèmes d'accessibilité, les résolutions des congrès organisés depuis 2000 . Les documents des congrès précédents ont été analysés sur la base de sources secondaires: Holzer Anton, Schwegler Barbara, 1998, "The Südtiroler Volksparte", dans De Winter Lieven, Türsan Huri (eds.), Regionalist parties in Western Europe, Routledge, London and New York, pp. 158-173. ; Caramani, D. and Y. Mény (eds.), Challenges to Consensual Politics: Democracy, Identity, and Populist Protest in the Alpine Region, Brussels, P.I.E.-Peter Lang, 2005 ; Pallaver G., The Südtiroler Volkspartei : from Irredentism to Autonomy, in De Winter L., Gómez-Reino M., Lynch P. (eds.), Autonomist Parties in Europe: Identity, Politics and the Revival of the Territorial Cleavage (ICPS, Bd. 2), Barcelona, Aleu, S.A., 2006, pp. 161-188 ; Pallaver G., "South Tyrol: from an ethnic to a new territorial cleavage", Paper presented at the 5th ECPR General Conference, 10-12 September 2009, Potsdam, Germany. Vu le nombre important de documents, ils ne sont pas listés en annexe mais peuvent être obtenus sur demande auprès des auteurs. 


\section{Territoire, ethnie et langue : le clivage centre-périphérie en Italie}

Selon la littérature ${ }^{27}$, les variables caractérisant la différentiation culturelle des communautés considérées sont les suivantes : la présence d'une ou de plusieurs langues minoritaires, le degré de diffusion de la langue minoritaire à l'intérieur du territoire régional, la distribution de la population parlant la langue minoritaires et sa localisation par rapport aux zones urbaines ou rurales, le statut officiel de la langue, la présence de référentiels culturels liés à la mémoire d'un passé autonome ou de mythes et traditions communes, le statut "international” de la langue minoritaire et les caractéristiques de l'image véhiculée par cette langue.

En Trentin-Haut-Adige, deux groupes ethnolinguistiques séparés habitent deux territoires distincts 28 : les italophones résident majoritairement dans la province de Trento, tandis que les deux tiers des habitants de la province de Bozen sont germanophones. Il existe également dans le Bozen une minorité parlant le ladin 29. En Vallée d'Aoste, en revanche, deux groupes ethnolinguistiques partagent le même territoire régional, entremêlés ${ }^{30}$. En Sardaigne, il existe un seul groupe ethnolinguistique, parlant le sarde aussi bien que l'italien - en raison notamment du processus d'italianisation forcée de la population mis en ouvre pendant les années cinquante et soixante ${ }^{31}$.

En ce qui concerne le degré de diffusion de la langue minoritaire à l'intérieur du territoire régional, avec environ un million de locuteurs (81\%), le sarde constitue la minorité linguistique la plus large d'Italie. En Vallée d'Aoste, le nombre d'habitants parlant couramment le français en tant que langue maternelle (aussi sous sa version locale, le franco-provençal) fut estimé à un peu moins de $17 \%$ en 2001 32, tandis qu'en 1980 ce groupe représentait

27. Caciagli M., Regioni d'Europa. Devoluzioni, regionalismi, integrazione europea, Bologne, Il Mulino, 2006 ; Melucci A.et Diani M., Nazioni senza stato. I movimenti etnico-nazionali in Occidente, Milan, Feltrinelli, 1992.

28. Di Sotto N., Dalla periferia all'Europa. I partiti etnoregionalisti e l'Unione Europea, Soveria Mannelli, Rubbettino, 2009.

29. Le ladin est une langue romane proche du frioulan parlé dans le Frioul et du romanche parlé en Suisse (dans le canton des Grisons). Elle est parlée en Haut-Adige, dans le Frioul et dans la province de Belluno en Vénétie. Au Trentin-Haut-Adige, le ladin est aujourd'hui parlé par environ $4 \%$ de la population. Voir les statistiques produites par le Landesinstitut für Statistik en 2006, 2007, 2008 ; Pallaver G., "South Tyrol From an Ethnic to a New Territorial Cleavage”, paper présenté à la Cinquième Conference Générale de l'ECPR, 10-12 Septembre, Potsdam, Allemagne, 2009).

30. Omezzoli T., "Lingue e identità valdostana », in Woolf S. (ed), Le Regioni dall'Unità ad oggi, La Valle d'Aosta, Turin, Einaudi, 1996, pp. 137-202 ; Cuaz M., " La Valle d'Aosta. Un'identità di frontiera tra Italia, Europa ed etnonazionalismi », in Nevola G., op. cit., pp. 118.

31. Accardo A., "Sardegna : le inquietudini di una regione autonoma », in Nevola G. (ed), op. cit., pp. 107-131 ; Pala C., «La sopravvivenza prima di tutto : voti ed eletti di due partiti etnoregionalisti in Sardegna e in Bretagna », Quaderni dell'Osservatorio Elettorale, 60, 2008, pp. 542.

32. Fondation Emile Chanoux, Une Vallée d'Aoste bilingue dans une Europe plurilingue, Aoste, Tipografia Valdostana, 2001. 
$58 \%$ de la population totale ${ }^{33}$. En Sud-Tyrol, $69 \%$ de la population parlait allemand, $27 \%$ l'italien et $4 \%$ appartenait au groupe linguistique ladin ${ }^{34}$.

Quant au statut de la langue à l'intérieur de la région et par rapport au niveau national, les minorités linguistiques sont protégées par des « mesures spéciales " prévues par l'article 6 de la Constitution, bien que cette norme opère une distinction entre différents degrés de protection. Le critère d'allocation de ces formes de protection est principalement territorial : les droits spéciaux sont liés au territoire où chaque minorité est concentrée, plutôt qu'aux individus parlant la langue minoritaire. Une distinction s'opère entre " minorités hyper-protégées » (les germanophones au Sud-Tyrol et les francophones en Vallée d'Aoste, ainsi que les slovènophones en Frioul) et «minorités reconnues ", c'est à dire l'ensemble des autres groupes ${ }^{35}$. Dans ces deux cas, la langue minoritaire bénéficie d'un statut de langue co-officielle au niveau régional (garanti par les chartes constitutionnelles régionales) et est formellement reconnue par les normes constitutionnelles nationales. En Sardaigne, la langue minoritaire est simplement protégée par des lois ordinaires. Les normes d'application sont régionales, comme la loi 26/1997 qui donne au sarde le statut de « deuxième » langue officielle avec l'italien sur le territoire de la région, et la loi nationale 482/1999, qui prévoit entre autre l'enseignement du sarde à partir de l'école primaire.

En revanche, la typologie de bilinguisme présent au Haut-Adige est très différente de celle présente en Vallée d'Aoste. Le particularisme valdôtain a un caractère "d'inclusion ", car les populations italophones, francophones et celles parlant le franco-provençal sont étroitement entremêlées et l'identité du groupe ethnolinguistique francophone est basée sur un sentiment d'appartenance communautaire et civique. Contrairement à la région du Haut-Adige où le statut d'autonomie établit une distinction entre les groupes linguistiques allemand et italien (mais aussi ladin) et où l'utilisation d'une langue ou de l'autre est obligatoire selon la langue maternelle du citoyen concerné, la Vallée d'Aoste reconnaît au français et à l'italien le statut de langue officielle. Ceci a entraîné la création d'un système éducatif parfaitement bilingue et le développement progressif d'un système de protection de la langue française très articulé.

En Trentin-Haut-Adige, le bilinguisme est en vigueur dans la province de Bozen (entre allemand et italien), mais il existe également une subdivision de la population sur la base de l'identité ethnique. La structure institutionnelle de

33. Région Autonome Vallée d'Aoste, Aperçu synthétique, Aosta, Bureau de Presse de la Présidence du Gouvernement, 1990.

34. Palermo F., “Asymmetric, 'Quasi-Federal' Regionalism and the Protection of Minorities. The Case of Italy”, in Tarr A.,Williams R. et Marko J. (ed), Federalism, Sub-National Constitutions, and Minority Rights, Londres, Greenwood Praeger, 2004, pp. 107-131.

35. Palici di Suni Prat E., Intorno alle minoranze, Turin, Giappichelli, 1999, pp. 29-33. 
la province est fondée sur une stricte séparation des deux groupes, italien et allemand, et sur la règle de «proportion ethnique » ou de « gouvernance de division ethnique 36 », tant en termes d'allocation des ressources économiques et des postes dans l'administration publique qu'en termes d'enseignement des langues. Le statut donne au troisième (plus petit) groupe, le Ladin, des garanties de représentation dans le parlement provincial. La situation au Sud-Tyrol peut donc être qualifiée de "particularisme d'exclusion ${ }^{37}$ ».

En Haut-Adige, la population italophone se concentre presque entièrement dans une vallée spécifique, la Vallée d'Adige, ainsi que dans la ville de Bozen. La population germanophone, majoritaire, se répartit aussi bien dans les campagnes que dans les villes, mais la population rurale est surtout germanophone, les italophones y résidant rarement. En Vallée d'Aoste, la population parlant le français et/ou le franco-provençal se situe largement dans les vallées latérales et les zones rurales ${ }^{38}$. En Sardaigne, en revanche, la population parlant le sarde se répartit en moyenne de façon assez régulière entre zones urbaines et rurales, bien que les populations rurales soient principalement sardophones.

La présence de références culturelles liées à la mémoire d'un passé autonome joue un rôle très important seulement dans deux des trois cas de mobilisation ethnolinguistique. Si la présence de mythes et traditions communs liés à la langue constitue un élément fondateur des identités culturelles des trois groupes, la mémoire historique liée à la présence d'institutions autonomes dans le passé fait partie des identités valdôtaine et sarde, mais pas de l'identité culturelle sud-tyrolienne ${ }^{39}$. D'une part, le paradigme identitaire valdôtain a toujours été fondé sur une présumée aspiration à la liberté et à l'autogouvernement qui a débuté en 1191 avec la "Charte de Franchises", pacte entre le comte Thomas $1^{\text {er }}$ de Savoie et les citoyens d'Aoste, qui octroyait à ces derniers une certaine autonomie, notamment en termes fiscaux. De même, la mémoire historique des Stamenti, les institutions représentatives de type parlementaire que les occupants aragonais avaient octroyées à la population sarde à partir du XVe Siècle et qui fonctionnèrent jusqu'à l'arrivée des Savoie, constituent une des bases de l'identité culturelle du peuple sarde.

En terme de position géographique du groupe minoritaire par rapport à ses «voisins » ethniques ${ }^{40}$, on peut distinguer d'une part les « îles ethniques » comme la Sardaigne, où le groupe ethnolinguistique minoritaire ne dispose d'aucune contrepartie dans d'autres pays; et d'autre part les « franges natio-

36. Palermo F., op. cit., p. 116.

37. Buisson D., Val d'Aoste et Haut-Adige : genèse et développements de deux particularismes en Italie, Mémoire non publié, IEP Rennes, 2000, p. 58.

38. Janin B., La Vallée d'Aoste. Tradition et Renowveau, Aoste, Musumeci Editeur, 1991.

39. Di Sotto N., op. cit.

40. Melucci A. et Diani M., op. cit. 
nales », comme le Haut-Adige et la Vallée d'Aoste, où le groupe minoritaire dispose d'une contrepartie dans la communauté linguistique dominante habitant l'État adjacent. En revanche, le statut « international » de la langue minoritaire varie significativement selon le cas. La protection du français et du sarde relève exclusivement du droit interne italien, tandis que la question $\mathrm{du}$ droit à l'autodétermination de la communauté germanophone en Haut-Adige a été intégrée dans le traité de paix de Paris de 1947. La résolution 1497 de l'Assemblée Générale des Nations Unies (1960) poussa l'Italie à adopter le nouveau statut d'autonomie de 1972. De plus, la présence d'une "mère patrie » a été fondamentale dans la formation de l'identité culturelle du SudTyrol, étant donné que l'Autriche a toujours été reconnue comme son «État garant » en acquérant une fonction protectrice. L'Autriche est intervenue à maintes reprises dans les négociations entre la communauté germanophone et l'État italien dans les années cinquante et soixante.

Constituant une «île ethnique », le groupe ethnolinguistique sarde n'a pas bénéficié de l'aide d'une « mère patrie ». En revanche, si l'armée et les services secrets français (et le général de Gaulle) se sont intéressés initialement à la possibilité d'annexer la Vallée d'Aoste à la France ${ }^{41}$, l'idée fut abandonnée suite à l'opposition de Truman et du commandement allié en Italie du Nord pendant le printemps 1945. A partir de 1946, la France se désintéressa complètement de la région.

Concernant l'image véhiculée par la langue minoritaire, les politiques d'italianisation développées à plusieurs reprises pendant le fascisme (dans les trois cas considérés), après la deuxième Guerre mondiale (en Sardaigne) ainsi que les politiques linguistiques régionales, ont eu pour conséquence l'utilisation de l'allemand aussi bien par les élites que par le peuple, tandis que le sarde et le franco-provençal sont principalement parlés par le peuple ${ }^{42}$.

La mobilisation ethnolinguistique en Vallée d'Aoste et en Sardaigne est principalement élitaire, tandis qu'au Haut-Adige il s'agit d'un phénomène populaire. Au Sud-Tyrol, le réseau associatif qui s'organise autour du SVP, comme le syndicat paysan Bauernbund, les associations d'agriculteurs, le syndicat ouvrier ASGB, les associations culturelles comme Heimatvereine, intè-

41. Nicco R., Le parcours de l'autonomie, Aoste, Musumeci Editeur, 2005.

42. Lengereau M., «La minorité ethnique valdôtaine de 1861 à nos jours : continuité et mutations ", in Sanguin A.-L., Les minorités ethniques en Europe, Paris, L'Harmattan, 1993, pp. 207-219; Accardo A., op. cit. ; Di Michele A., Palermo F. et Pallaver G. (ed), Fine di un conflitto. Dieci anni dalla chiusura della questione sudtirolese, Bologne, Il Mulino, 2003 ; Roux C., «Des difficultés de la contestation peripherique dans le Mezzogiorno. L’example du Parti Sarde d'Action ", Pôle Sud, 20, 2004, pp. 147-164 ; Roux C., “The Partito Sardo d'Azione: regionalist mobilization in Southern Italy”, in De Winter L., Gómez-Reino Cachafeiro M. et Lynch P. (ed), Autonomist parties in Europe: Identity, Politics, and the Revival of the territorial Cleavage, Barcelone, Institut de Ciències Polítiques i Socials, 2006, pp. 189-218. 
gre l'entière communauté germanophone ${ }^{43}$. En revanche, en Vallée d'Aoste, à partir de 1948, les acteurs de la mobilisation ethnolinguistique sont restés plus ou moins les mêmes et se sont limités au clergé et à l'élite bourgeoise urbaine ${ }^{44}$. Les initiatives de défense de l'identité valdôtaine sont en majorité organisées par un petit cercle cultivé 45 lié aux associations culturelles et linguistiques (Institut Régional de la Résistance, Fondation Chanoux, Centre d'Etudes franco-provençales, etc.) et par le syndicat valdôtain (SAVT, Syndicat autonome valdôtain des travailleurs). En Sardaigne, outre l'action des élites intellectuelles et les initiatives des mouvements culturels de défense $\mathrm{du}$ sarde, les seuls acteurs de la mobilisation ethnolinguistique sont les partis et les mouvements ethnorégionalistes ${ }^{46}$.

C'est donc seulement au Sud-Tyrol que la langue a toujours été le principal facteur de différentiation culturelle de la minorité ethno-territoriale germanophone. L'identité linguistique et l'ethnie sud-tyrolienne sont vécues comme des expériences totalisantes par le groupe germanophone. L'absence d'une mémoire historique commune d'autonomie, la présence de formes de protection internationale de la langue, les règles de «proportion ethnique », le système éducatif séparé au niveau régional et le compartimentage complet des deux communautés linguistiques font de la langue l'élément central de l'identité culturelle sud-tyrolienne.

L'identité valdôtaine est, elle, bâtie sur la langue mais également sur d'autres éléments identitaires, dont la mémoire d'un passé d'autonomie. Le caractère «d'inclusion » du particularisme valdôtain réduit le pouvoir cohésif de la langue à l'intérieur du groupe ethnolinguistique francophone en Vallée d'Aoste. Si la langue reste un élément fondamental du sentiment de l'identité valdôtaine, son rôle de facteur cohésif du groupe ethnolinguistique valdôtain est aujourd'hui moins significatif qu'il y a cinquante ans. En Sardaigne, l'importance de la langue en tant que vecteur identitaire a été beaucoup plus grande par le passé qu'aujourd'hui : pendant la domination aragonaise puis piémontaise, le sarde était considéré comme facteur de mobilisation révolutionnaire et ethnique du peuple ${ }^{47}$. Après 1945 , la mobilisation ethno-territoriale en Sardaigne s'est développée principalement autour des dimensions institutionnelles et socio-économiques ${ }^{48}$. La situation changea seulement à partir des années soixante-dix, quand les mouvements indépendantistes et autonomistes utilisèrent la langue sarde, non protégée et non enseignée dans la région, comme principal vecteur identitaire.

43. Buisson D., op. cit., pp. 72.

44. Omezzoli T. op. cit.

45. Buisson D., op. cit., p. 73.

46. Roux C., op. cit.; Pala C., op. cit.

47. Petrosino D., "National and regional movements in Italy: the case of Sardinia", in Coakley J. (ed), The social origins of nationalist movements, Londres, Sage, 1992, pp. 124-146.

48. Pala C., op. cit. 
Les trois acteurs de la mobilisation ethno-territoriale sélectionnés montrent des parcours très différenciés en ce qui concerne les modalités et les motivations à l'origine de leur création. Les trois partis représentent, à l'intérieur des systèmes respectifs partisans régionaux, les principaux partis ethnorégionalistes actifs sur leur territoire. Ils ont modifié leurs stratégies électorales et leurs systèmes d'alliances à maintes reprises pendant les cinquante dernières années, à l'intérieur d'un cadre stratégique « d'adaptation » au contexte de la compétition électorale et sur la base de certains choix stratégiques en termes de politiques publiques ${ }^{49}$. Nous mobilisons ici plusieurs extraits de programmes électoraux des trois partis tout au long de la période considérée. Nous les avons sélectionnés en fonction de leur représentativité par rapport à notre objet d'analyse, et notamment en ce qui concerne l'évolution de l'idéologie des trois partis. Nous avons donc identifié les différentes positions idéologiques que les trois forces politiques ont adoptées pendant la période de temps intégrée dans l'analyse. Nous en présentons en outre des exemples à travers des extraits des documents analysés ${ }^{50}$. Le discours politique des partis considérés est décliné ici sur la base des trois dimensions de territoire, ethnie et langue, afin de voir comment ces dernières réussissent (ou non) à devenir les aspects centraux des structures idéologiques et de la stratégie partisane.

La notion de territoire peut se référer à la fois aux espaces géographiques dans lesquels la minorité ethnolinguistique réside, aussi bien qu'au concept plus politique de corps politique, et donc aux frontières du système politique infra-étatique par rapport à celles imposées par le pouvoir central. Le territoire reste un élément fondamental de toute mobilisation ethnolinguistique et identitaire des partis ethnorégionalistes considérés. Si le SVP parle du SudTyrol comme «l'espace naturel de notre communauté [...], il faut se séparer de Trento, car ce n'est pas une question régionale [de la Région administrative du Trentin-Haut-Adige] mais c'est notre nécessité de donner à la Province [de Bolzano/Bozen] le même degré [de pouvoir autonome] de la Région» et de la "protection de la zone alpine en tant que lieu [de résidence] du peuple SudTyrolien 51 ", le PSdAZ considère depuis toujours l'île " comme un patrimoine à préserver et à défendre, la terre des nos ancêtres dans laquelle nous voulons

49. Dandoy R. et Sandri G., «I programmi elettorali dei partiti regionalisti europei : un'analisi comparata ", Quaderni dell'Osservatorio Elettorale, 59, 2008, pp. 63-94.

50. Ces extraits de programmes électoraux sont juxtaposés ici sans entrer toutefois dans le détail du contexte de l'élection spécifique, car ils sont analysés en termes de représentation symbolique et de substantivation des identités du parti et non par rapport à la stratégie électorale inhérente à l'élection correspondante. Par exemple, les programmes électoraux des élections législatives de 1976 et des élections régionales de 2009 sont ici considérés comme équivalents aux fins de l'analyse. Le discours analysé, pourtant, n'est pas considéré comme atemporel, mais au contraire dans son évolution dans le temps, tout en excluant une analyse approfondie du contexte lié à l'élection correspondante.

51. Südtiroler Volkspartei, Programme électoral, Élections provinciales 2008. 
faire vivre nos fils 52 ». Le PSdAZ met en exergue le fait qu'il faut «libérer la Sardaigne des toutes bases militaires italiennes de l'OTAN, qui ont appawvri le territoire, [cet objectif est le] début et [la] fin de notre combat 53 ».

Si l'on considère le territoire en tant qu'espace politique et institutionnel, les positions des différents partis sur l'évaluation du statut de décentralisation varient significativement : le territoire devient un lieu tangible où exercer les droits à l'auto-détermination des peuples. L'UV demande la mise en place d'un « régionalisme différencié contre le fédéralisme factice proclamé et le véritable centralisme pratiqué » par l'État central, jusqu'à arriver, plus radicalement, à la demande de "modification de l'art. 116 de la Constitution [qui] doit garantir la nature du pacte de l'entente pour la modification de notre statut spécial 54 ». Pour l'UV il faudra «lutter contre les initiatives évidentes ou dissimulées de diminuer le rôle historique et actuel des autonomies spéciales » et " accélérer le transfert de compétences et de financements relatifs à notre région $55 »$.

Lors de son congrès de 2003, l'UV surveille le travail sur la réforme de l'organisation territoriale de l'État, du gouvernement et du parlement national, pour préserver et élargir "nos prérogatives autonomistes afin que notre région puisse toujours faire entendre sa voix $56 »$. Le PSdAZ considère la possibilité d'arriver à une "pleine indépendance de la Sardaigne, dans le cadre d'une Europe formée de peuples et de régions sans les contraintes imposées par les faux États-Nation comme l'Italie 57 ", tandis que le SVP veut la garantie d'une encore "plus grande autonomie de la Province ", étant donné qu'il faut tout d'abord "s'occuper de notre territoire, pas forcément dans un véritable projet fédéraliste de l'État italien» (Südtirol Handbuch 2004).

Le concept d'ethnie a également été utilisé de façon différente par les partis étudiés. Le leadership des trois partis s'est bâti sur une image d'uniques défenseurs de l'identité communautaire sur la base d'une combinaison précise d'éléments constituant l'identité ethnique du groupe. Dans les statuts des trois partis, on revient souvent sur la notion de peuple, en tant que communauté partageant un sentiment d'appartenance. On est passé d'une interprétation exclusive du terme à une interprétation inclusive. Ceci concerne surtout le SVP mais également, en un sens, l'UV, pour qui le mot peuple marque la limite entre «nous » et « eux». Dans le discours politique des années cinquante et soixante du SVP, les italophones sont considérés comme des citoyens parlant une langue différente, mais aussi appartenant à une ethnie différente.

52. Partito Sardo d'Azione, Programme électoral, Élections régionales 2009.

53. Partito Sardo d'Azione, Programme électoral, Élections régionales 2004.

54. Union Valdôtaine, Programme électoral, Élections législatives 2008.

55. Union Valdôtaine, Programme électoral, Élections législatives 2006.

56. Union Valdôtaine, Congrès national de 2003, Rapport du Président du Parlement Régional.

57. Partito Sardo d'Azione, Congrès national de 2004, Rapport final. 
Un document programmatique de l'UV de 1976 soulignait ainsi qu' " an moment où dans le monde entier les ethnies et les peuples aujourd'bui minoritaires sont en train de seconer le joug des ethnies dominantes et des étatsnation, la Vallée d'Aoste ne peut pas manquer de suivre ce même chemin. En effet, les Valdôtains sont en train eux aussi de prendre conscience de leur état d'aliénation culturelle et linguistique et de colonisation économique auxquelles l'État italien les a conduits par une oppression tantôt brutale, tantôt sournoise, mais toujours efficace 58 ». Le concept d'ethnie n'était alors pas encore mobilisé par l'idéologie unioniste. La résolution finale du congrès de l'UV de 1993, en revanche, demande "la définition de l'identité ethnique, linguistique et culturelle du peuple valdôtain 59 ».

Par ailleurs, le concept de groupe ethnique séparé est devenu depuis les années quatre-vingt la caractéristique principale des programmes politiques de l'UV. Le SVP considère l'ethnie allemande comme " celle de notre peuple, l'origine de nos gens et la possibilité de notre rachat $60 »$. En fait, ce parti a utilisé largement l'opposition entre « les Italiens » et «nous » dans la construction de son identité. Sans l'exploitation idéologique de cette tension identitaire, le SVP, en tant que parti défenseur de l'identité ethnique, se serait rapidement effondré. De plus, la question ethnique apparaît strictement liée à la question religieuse. Bien que la dimension religieuse ne soit pas comparable au contexte nord-irlandais, le parti sud-tyrolien a conquis sa place dans la société régionale en montrant qu'il était un parti qui allait «unir la question de la différence ethnique avec une très forte appartenance à la religion catholique » et qui se dotait «d'une vision chrétienne-bumaniste de son peuple et de l'bumanité en général $61 »$.

Le PSdAZ a toujours intégré la notion de peuple sarde à celle de nation sarde. C'est dans cette correspondance conceptuelle que résident les principaux instruments à développer pour prendre conscience d'une condition de subordination : "la vraie solution c'est de se percevoir finalement comme un peuple. [...] Quand on a élaboré le concept de la "constante de la Résistance sarde" 62 on voulait indiquer un peuple fier, qui lutte et qui pourra s'émanciper seul, sans besoin d'aide. Les Italiens ont toujours profité de notre peuple». Donc, «le peuple sarde a le droit d'affirmer sa souveraineté sur son propre territoire » et «le Parti Sarde d'Action [a le droit d'affirmer] l'existence de la nation sarde et le droit du peuple sarde à la liberté $63 »$.

58. Union Valdôtaine, Programme électoral, Élections législatives 1976.

59. Union Valdôtaine, Résolution finale du Congrès national, 1993.

60. Südtiroler Volkspartei, Résolution finale du Congrès, 2004.

61. Südtiroler Volkspartei, Résolution finale du Congrès, 2004.

62. Avec le terme «constante de la Résistance sarde» nous indiquons les difficultés historiques des différents envahisseurs de la Sardaigne à conquérir toute l'île. Ceci est devenu un refrain dans le discours politique des mouvements indépendantistes sardes et même du PSdAZ.

63. Partito Sardo d'Azione, Statuts du PSdAZ, art. 1 et 3. 
Les positions des partis considérés ont bien évolué dans le temps, en modifiant dans certains cas cette attitude idéologique d'exclusion pour intégrer des discours politiques plus inclusifs. L'UV, pour des raisons stratégiques, s'est ouvert aux Italiens des autres régions qui partagent les mêmes valeurs et objectifs politiques. Cette stratégie d'inclusion entendait s'allier les votes des habitants de la Vallée d'Aoste, quelles que soient leurs origines ethniques. En outre, les requêtes d'autonomie du parti sont de plus en plus basées sur la notion de défense du particularisme du peuple valdôtain plutôt que sur la défense des droits de la minorité linguistique francophone. C'est l'appartenance au peuple et à l'ethnie valdôtaine, comme communauté de valeurs et de traditions, qui est mise en exergue dans les derniers programmes du parti. Lors du congrès national de 2003, un ancien président du parti souligna l'ampleur du parcours idéologique de l'UV : «Le mouvement lui aussi s'est transformé : il est devenu un grand mouvement populaire, interprète des différentes instances dont la nouvelle communauté valdôtaine est porteuse. Un sens de l'autonomie, partagé par toujours plus de Valdôtains, un sens de l'appartenance plus diffusé, une conscience identitaire peut-être moins forte mais beaucoup plus répandue 64 ». Dans le passé, le Parti Sarde d'Action a eu, lui aussi, tendance à interpréter la notion d'ethnie en un sens exclusif. Pour réagir aux attaques des élites intellectuelles italiennes qui niaient l'existence d'une ethnie sarde séparée et d'un peuple sarde à part entière, les partis indépendantistes de l'île ont depuis longtemps opposé des argumentations en faveur de la construction d'une identité ethnique spécifique. Pendant les années soixantedix, des études scientifiques démontrèrent que les Sardes ne possédaient pas le même génotype que les autres Italiens ${ }^{65}$. Toutefois, il y eut une certaine évolution dans l'idéologie des acteurs de la mobilisation sarde, et du PSdAZ en particulier, qui contribua à développer une notion différente d'indépendantisme et d'autonomisme institutionnel, en vigueur encore aujourd'hui. Le PSdAZ, ainsi, "agit pour réaliser la pleine reconnaissance du peuple sarde auprès des autres peuples d'Europe 66 ». A partir de ce moment, le PSdAZ liera toujours son engagement politique à des repères ethniques, avec ces slogans : "on naît tous égaux, mais après nous on devient Sarde", "peuple sarde, peuple d'Europe ", "nous avons une culture à part donc nous constituons un peuple à part».

En ce qui concerne le SVP, en revanche, le rôle de l'ethnie dans les repères identitaires a été plutôt stable, comme on l'a déjà souligné. De plus, dans le cas du SVP, le concept d'ethnie est strictement lié à celui de langue, tant dans la définition du paradigme idéologique du parti que dans l'identification de ses objectifs politiques. Le SVP s'est toujours basé sur la dichotomie ItaliensAllemands (mieux, germanophones) pour définir la communauté germanophone du Sud-Tyrol en tant qu'ethnie et en tant que peuple. Le parti répète

64. Union Valdôtaine, Congrès national de 2003, Rapport de Charles Perrin.

65. Salvi S., Nazioni proibite, Florence, Vallecchi, 1973, pp. 23-26.

66. Partito Sardo d'Azione, Résolutions finale du Congrès national de 1981. 
que «l'existence d'un peuple Sud-Tyrolien est à la base de la reconnaissance des aspirations » d'autonomie de la communauté germanophone 67 .

Si le poids relatif aux concepts de territoire et d'ethnie peut varier sensiblement dans le temps (et selon les cas) au sein des statuts et des programmes électoraux des trois partis considérés, ce qui reste constant est le rôle de la langue en tant que vecteur identitaire du groupe ethnolinguistique. Les partis semblent conscients du fait que la langue est perçue comme un élément identitaire facilement reconnaissable et très proche des citoyens et, donc, plus efficace en termes de mobilisation.

L'État central a à maintes reprises empêché (à travers l'annulation de plusieurs lois régionales) que la Sardaigne se dote de normes législatives spécifiques pour la protection de la langue sarde dans son statut d'autonomie, comme c'est le cas dans la Vallée d'Aoste et de la province de Bolzano/Bozen. L'adoption de normes législatives nationales et régionales de protection de la langue minoritaire et la mise en ouvre, dans le temps, de lois d'application de l'autonomie régionale en termes d'enseignement et de bilinguisme, ont amené l'UV et le SVP à atténuer leurs requêtes concernant la langue, tandis que le PSdAZ renforçait le rôle de la langue comme objectif politique.

Par ailleurs, si les équilibres démographiques restent assez stables au SudTyrol (la population germanophone est passée de $76 \%$ du total de la population du Sud-Tyrol en 1921, à 69 \% en 2001) et en Sardaigne, en revanche l'immigration italienne a augmenté considérablement au cours des trente dernières années en Vallée d'Aoste, transformant le paysage linguistique de la région. La langue reste toujours un élément important pour l'UV dans le choix de ses alliances électorales : " [on s'est allié au centre-gauche car] son programme est plus respectueux des principes de l'autonomie régionale et des problématiques des minorités linguistiques 68 ». A coté d'une menace de « disparition » due aux nouvelles populations immigrées, la langue a retrouvé dernièrement dans l'idéologie de l'UV son rôle d'objectif politique de première importance. Selon le parti, "les jeunes doivent connaître nos langues : le français, le francoprovençal et les dialectes germanophones 69 ».

Les politiques des gouvernements régionaux guidés par l'UV s'orientent, enfin, vers un renforcement de l'enseignement et de la protection du patois franco-provençal, comme le démontrent les lois régionales sur l'enseignement obligatoire du patois dans les écoles primaires adoptées entre 2005 et 2008. Selon l'UV, les valdôtains sont à la base d'une vraie "civilisation valdôtaine » qui "appartient naturellement à la grande civilisation des Alpes et au vaste monde de la francophonie [...]. Il est pour nous fondamental de maintenir et

67. Autonome Provinz Bozen, Südtirol Handbuch, 2004.

68. Union Valdôtaine, Programme électoral, Élections législatives 2006.

69. Union Valdôtaine, Programme électoral, Élections régionales de 2003. 
valoriser les caractéristiques particulières $d u$ peuple valdôtain. Tout d'abord, le sens de la communauté et de l'appartenance, la défense intransigeante de nos particularités linguistiques 70 ".

Les revendications sud-tyroliennes, en revanche, visent à « conserver ce qui existe. Il faut tout d'abord veiller à ce que les conditions d'application $d u$ statut légal de l'allemand à l'école et dans l'administration publique soient respectées 71 ». Néanmoins, le SVP souligne que «l'allemand est au cour de notre identité et tout notre espoir [...] Donc, il faut garder un haut niveau d'attention par rapport à la langue [...]. Tous les efforts que nous avons mis en ceuvre pour acquérir ce dont nous disposons aujourd'hui [en termes de bilinguisme et de protection de l'allemand] il faudra les renouveler pour maintenir ces avancées : la langue est comme nos montagnes : il faut la respecter et l'utiliser correctement sinon elle va nous punir $72 »$.

Le SVP consolide donc ses objectifs politiques autour de l'obtention d'une autonomie institutionnelle accrue là où la langue possède une place bien définie. Lors des élections de 2008, le programme électoral du parti demandait une autonomie plus avancée, avec une présence beaucoup plus limitée de l'État central sur le territoire sud-tyrolien et des mesures visant aussi l'autonomie linguistique, comme le transfert de la section régionale de la télévision d'État sous le contrôle de la province, pour ce qui concerne la langue.

La condition de langue non protégée et la situation de possible disparition a mené le PSdAZ à reconsidérer la langue comme un aspect indispensable de son engagement politique. Le sarde était une langue très répandue, utilisée quotidiennement par la population entière de l'île. A partir des années soixante, le sarde est menacé de disparition, connaissant une période qu'on peut définir de honte linguistique ${ }^{73}$, durant laquelle l'utilisation quotidienne de la langue sarde au sein des familles décroît pour des raisons principalement socio-économiques, liées à l'ancrage de l'italien (en raison d'une aspiration à la «modernité »), et dans un contexte où l'État central essaye à plusieurs reprises de mettre en œuvre de mesures pour décourager l'utilisation du sarde dans les écoles et l'administration publique. En fait, le PSdAZ, lors de sa fondation, ne mettait en exergue que de façon marginale les questions culturelles et linguistiques. Jusqu'aux années soixante-dix, le Parti n'a pas soulevé le problème linguistique ni celui de la tutelle du sarde, tout simplement parce que le problème n'existait pas.

A partir des années soixante-dix, pour répondre à la fois à une nécessité réelle de défense de la langue et aux autres partis indépendantistes qui avaient

70. Union Valdôtaine, Programme électoral, Élections régionales de 1998.

71. Südtiroler Volkspartei, Programme électoral, Élections provinciales de 2008.

72. Südtiroler Volkspartei, Résolutions finale du Congrès national de 2004.

73. Pala C., op. cit. 
déjà mis en avant l'objectif politique de la langue sarde, le PSdAZ a commencé à intégrer cette dimension dans son paradigme idéologique. La langue est devenue aujourd'hui un point central dans tous les programmes électoraux du PSdAZ et aussi dans les initiatives législatives du Parti au sein des institutions régionales et provinciales. Plusieurs articles des statuts du parti affirment en fait que la langue sarde représente «l'expression de la conscience nationale $d u$ peuple sarde et donc [le PSdAZ] travaille pour sa diffusion, sa valorisation et son utilisation officielle auprès des écoles et des institutions ${ }^{74}{ }^{\prime}$. Pour le parti, il faut absolument «sortir d'une condition terrible dans laquelle les Italiens nous refoulent: celle de la destruction de notre langue, ancienne, dérivée directement du latin et de la langue, encore mystérieuse, de nos grands ancêtres, les nuragiques [...] Il faut arrêter ça, le parti doit s'engager encore plus dans le combat pour la langue. [...] Le parti peut devenir le seul acteur social et institutionnel qui va défendre la langue sarde face à ceux qui veulent la détruire 75 ».

\section{Succès (partiels) de la mobilisation}

Cette étude a tenté de répondre à deux principales questions de recherche. D'une part, le rôle de la langue en tant que vecteur identitaire dans les communautés linguistiques minoritaires sélectionnées a été analysé. D’autre part, l'étude a essayé d'identifier le rôle de la langue comme objectif politique des partis ethnorégionalistes censés représenter des minorités linguistiques spécifiques. En ce qui concerne la première question de recherche, on peut affirmer que dans les trois cas sélectionnés, la langue a représenté, à un moment ou un autre de l'histoire de la mobilisation ethnolinguistique régionale, un vecteur identitaire fondamental pour assurer la cohésion du groupe mobilisé. Toutefois, l'intensité du renforcement de la cohésion interne du groupe mobilisé sur le territoire exercée par la langue varie sensiblement selon les cas et, au sein du même groupe ethnolinguistique, selon les périodes. Parmi les trois cas sélectionnés, le seul où le facteur linguistique représente, de façon assez stable dans le temps, le principal élément de différentiation culturelle est le cas du Sud-Tyrol. Le rôle de la langue comme vecteur identitaire reste central au Sud-Tyrol, il est moins significatif en Vallée d'Aoste (au moins récemment), et relativement faible en Sardaigne (au moins pendant certaines périodes de son histoire).

Deuxièmement, le poids de la langue comme objectif politique a été examiné par rapport aux autres éléments idéologiques distinctifs des partis ethnorégionalistes en tant qu'entrepreneurs ethniques : ethnie et territoire. Le territoire représente toujours un objectif politique de premier ordre tant pour le PSdAZ que pour le SVP et l'UV. Si le premier se mobilise aujourd'hui pour

74. Partito Sardo d'Azione, Statuts du PSdAZ, article 3.

75. Partito Sardo d'Azione, Programme électoral, Élections régionales 2004. 
achever l'indépendance de la région, les deux autres partis demandent la mise en œuvre de formes d'autonomie politique et institutionnelle encore plus poussées. Si dans l'idéologie du SVP les concepts d'ethnie et de langue ont été toujours liés et recouvrent encore aujourd'hui une place centrale, dans le cas de l'UV le concept d'ethnie a récemment (depuis les années quatre-vingt) remplacé celui de langue dans le paradigme identitaire du parti. En Sardaigne, la mobilisation ethno-territoriale s'est développée sur la base de motivations principalement socio-économiques et de demandes de décentralisation au niveau institutionnel plutôt que culturel. La langue n'est devenue un objectif politique du PSdAZ que récemment, notamment depuis les années soixantedix.

En conclusion, le poids de la langue comme objectif politique des trois partis sélectionnés est lié à la position de chacune des trois langues minoritaires comme vecteur identitaire pour la cohésion du groupe mobilisé, et donc à l'évolution de la perméabilité des langues minoritaires à l'italien. Les trois cas peuvent être donc résumés de la façon suivante :

- Le cas de la Vallée d'Aoste : la perméabilité forte entre les trois langues néo-latines parlées dans la région (français, franco-provençal, italien standard) implique que le français et le franco-provençal se noient progressivement dans l'italien qui renforce son rôle de langue véhiculaire ${ }^{76}$. L'UV, suite aux importants changements démographiques des dernières décennies, a dû par conséquent se repositionner sur la question de la langue, en insistant plutôt sur l'élément du territoire dans son identité politique, se replier sur un nationalisme ethnique et se focaliser sur une acception de l'ethnicité fondée sur le sentiment d'appartenance à une communauté dotée d'une histoire et de traditions communes. Ce repositionnement est justifié par les importants moyens financiers alloués à la région et par la volonté de garantir cette manne financière pour tous les habitants de la Vallée quelle que soit leur origine.

- Le cas du Trentin-Haut-Adige : dans cette région, on ne trouve pas de perméabilité forte entre l'allemand et l'italien, vu que les deux communautés vivent complètement séparées. Dans cette situation, qui d'ailleurs est assez semblable au cas belge, les coûts d'apprentissage de l'autre langue sont élevés. Ceci a entraîné le maintien d'une frontière nette entre les deux groupes ethnolinguistiques (germanophones et italophones), d'autant plus que les structures éducatives de la région protègent la langue allemande depuis 1946. Par conséquent, étant donné que dans l'identité politique du SVP les concepts d'ethnie et de langue ont été toujours liés, cette dernière conserve encore aujourd'hui

76. En 1980, la moitié de la population indiquait le franco-provençal comme langue maternelle, tandis qu'en 2001 le pourcentage a chuté à 15,4\%. Moins de $2 \%$ de la population affirme utiliser couramment le français en famille. Fondation Emile Chanoux, Une Vallée d'Aoste bilingue dans une Europe plurilingue, Aosta, Tipografia Valdostana, 2001, p. 106. 
une place centrale dans l'idéologie du parti, bien que l'élément du territoire soit en train de renforcer son importance relative ${ }^{77}$.

- Le cas de la Sardaigne : en raison d'une situation de perméabilité moyenne entre le sarde et l'italien standard, le rôle de la langue en tant que vecteur identitaire de la minorité sardophone et en tant que cible de l'identité politique du PSdAZ, est resté assez constant depuis les années soixante-dix. Toutefois, à cause des pressions économiques et administratives exercées sur la société régionale, la langue sarde a connu récemment un déclin significatif dans l'usage quotidien en tant que langue véhiculaire. Cela a justement entrâné une réorientation du mouvement national sarde vers la défense de la langue afin d'éviter de rendre la revendication autonomiste caduque.

Donc, parmi les trois acteurs sélectionnés, le seul parti qui identifie de façon stable la défense de la langue minoritaire en tant qu'objectif politique prioritaire est le SVP, car il peut s'appuyer sur un électorat très stable démographiquement. De plus, la mise en œuvre progressive du processus de dévolution de compétences législatives du centre vers la périphérie a obligé le parti à radicaliser ses requêtes d'autonomie, en demandant entre autre plus de décentralisation culturelle, afin de justifier son existence. L'UV, qui d'une part a radicalisé ses requêtes en termes d'autonomie institutionnelle pendant les trente dernières années, a d'autre part modéré partiellement ses revendications en termes de décentralisation culturelle, vu que la mise en ouvre progressive du bilinguisme au sein des institutions administratives et éducative a limité le recours à certains thèmes à la base de sa mobilisation politique.

77. Pallaver G., “South Tyrol From an Ethnic to a New Territorial Cleavage”, Papier présenté à la $5^{\mathrm{e}}$ Conférence Générale de l’ECPR, Potsdam, Allemagne, 10-12 Septembre 2009 
ANNEXE 1. Performances électorales de I'UV, du SVP et du PSdAZ, 1948-2009.

Tableau 1 - Performances électorales de l'UV (en \% des voix exprimées en Vallée d'Aoste)

\begin{tabular}{|c|c|c|c|}
\hline & Européennes & Nationales & Régionales \\
\hline 1949 & & & 43.6 \\
\hline 1953 & & n.p. & \\
\hline 1954 & & - & 29.2 \\
\hline 1958 & & 51.1 & \\
\hline 1959 & & & 51.4 \\
\hline 1963 & & 51.2 & 20.4 \\
\hline 1968 & & 47.7 & 16.6 \\
\hline 1972 & & 47.9 & \\
\hline 1973 & & & 11.5 \\
\hline 1976 & & 32.1 & \\
\hline 1978 & & & 24.8 \\
\hline 1979 & 37.5 & 45.8 & \\
\hline 1983 & & 38.8 & 27.1 \\
\hline 1984 & 24.8 & & \\
\hline 1987 & & 55.2 & \\
\hline 1988 & & & 34.2 \\
\hline 1989 & 36.4 & & \\
\hline 1992 & & 49.6 & \\
\hline 1993 & & & 37.3 \\
\hline 1994 & 32.3 & 54.1 & \\
\hline 1996 & & 48.6 & \\
\hline 1998 & & & 40.1 \\
\hline 1999 & 45.9 & & \\
\hline 2001 & & 34.9 & \\
\hline 2003 & & & 47.2 \\
\hline 2004 & 37.5 & & \\
\hline 2006 & & 30.7 & \\
\hline 2008 & & 37.8 & 44.4 \\
\hline 2009 & 37.1 & & \\
\hline
\end{tabular}

Source : Ministère de l'Intérieur italien.

Note : n.p. : aucune liste est présentée par le parti.

Note : pour les élections nationales, dans les trois tableaux, on a présenté ici les voix pour l'élection de la Chambre des Deputés. 


\section{Tableau 2 - Performances électorales du SVP}

(en \% des voix exprimées en province de Bozen)

\begin{tabular}{|c|c|c|c|}
\hline & Européennes & Nationales & Provinciales \\
\hline 1948 & & 62.7 & 67.6 \\
\hline 1952 & & & 64.8 \\
\hline 1953 & & 60.0 & \\
\hline 1956 & & & 64.4 \\
\hline 1958 & & 60.8 & \\
\hline 1960 & & & 63.9 \\
\hline 1963 & & 56.6 & \\
\hline 1964 & & & 61.3 \\
\hline 1968 & & 58.5 & 60.7 \\
\hline 1972 & & 59.0 & \\
\hline 1973 & & & 56.4 \\
\hline 1976 & & 59.6 & \\
\hline 1978 & & & 61.3 \\
\hline 1979 & 62.1 & 62.9 & \\
\hline 1983 & & 59.6 & 59.4 \\
\hline 1984 & 63.1 & & \\
\hline 1987 & & 58.3 & \\
\hline 1988 & & & 60.4 \\
\hline 1989 & 53.0 & & \\
\hline 1992 & & 57.3 & \\
\hline 1993 & & & 52.2 \\
\hline 1994 & 57.0 & 60.178 & \\
\hline 1996 & & $28.0^{79}$ & \\
\hline 1998 & & & 56.6 \\
\hline 1999 & 56.0 & & \\
\hline 2001 & & $60.5^{80}$ & \\
\hline 2003 & & & 55.6 \\
\hline 2004 & 46.7 & & \\
\hline 2006 & & 53.4 & \\
\hline 2008 & & 44.3 & 48.1 \\
\hline 2009 & 52.2 & & \\
\hline
\end{tabular}

Source : Ministère de l'Intérieur italien.

78. Pourcentage de votes pour le SVP aux législatives de 1994 dans la partie proportionnelle, collège de Bozen.

79. Pourcentage de votes pour la coalition SVP-Popolari aux législatives de 1996 dans la partie proportionnelle, collège de Bozen.

80. Pourcentage de votes pour la coalition SVP-Ulivo aux élections législatives de 2001 dans la partie proportionnelle, collège de Bozen. 


\section{Tableau 3 - Performances électorales du PSdAZ}

(en \% des voix exprimées dans la région Sardaigne)

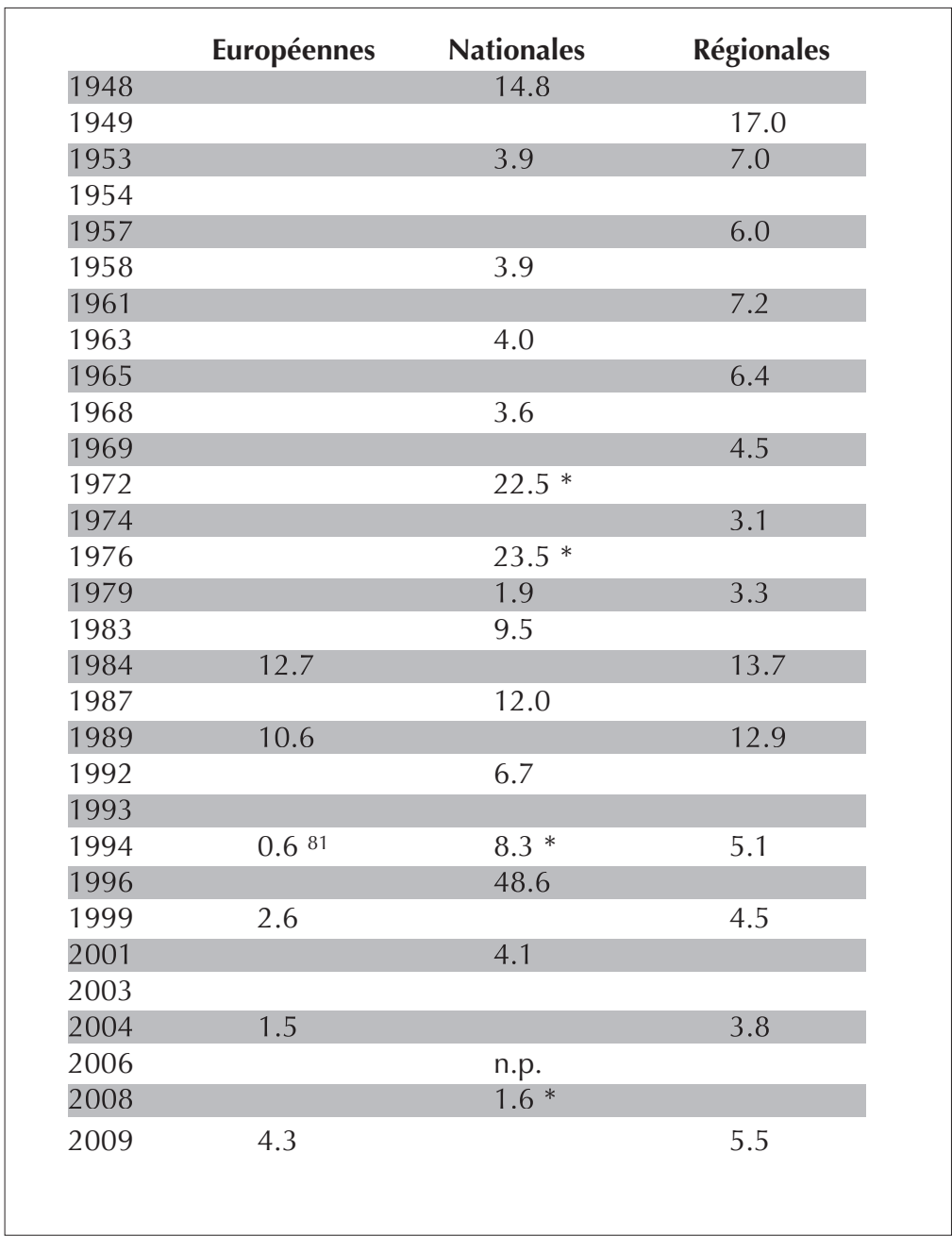

Source : Ministère de l'Intérieur italien.

* En alliance avec d'autres partis nationaux.

Note : n.p. : aucune liste est présentée par le parti.

81. Pour les élections de 1994 à 2009, la Sardaigne a été unifiée à la Sicile dans une circonscription électorale unique aux élections européennes, ce qui rend très difficile de décliner les résultats du PSdAZ sur une base régionale. Les résultats du PSdAZ semblent donc avoir chuté entre 1994 et les élections précédentes, mais cette impression est due au fait qu’à partir de 1994 les pourcentages sont calculés sur la base d'une circonscription qui intègre également la population de la Sicile. Pour l'instant, ceux-ci ne sont pas disponibles sur le site du Ministère de l’Intérieur des donnés agrégées au niveau de la Sardaigne. 